\title{
Acupuncture Treatment for Bortezomib-Induced Peripheral Neuropathy: A Case Report
}

\author{
Ting Bao, ${ }^{1,2}$ Ruixin Zhang, ${ }^{2}$ Ashraf Badros, ${ }^{1}$ and Lixing Lao ${ }^{2}$ \\ ${ }^{1}$ Marlene and Stewart Greenebaum Cancer Center, The University of Maryland School of Medicine, University of Maryland, \\ Baltimore, MD 21201, USA \\ ${ }^{2}$ Center for Integrative Medicine, The University of Maryland School of Medicine, University of Maryland, \\ Baltimore, MD 21201, USA \\ Correspondence should be addressed to Lixing Lao, llao@compmed.umm.edu
}

Received 31 October 2010; Accepted 17 January 2011

Academic Editor: Steve McGaraughty

Copyright (๑) 2011 Ting Bao et al. This is an open access article distributed under the Creative Commons Attribution License, which permits unrestricted use, distribution, and reproduction in any medium, provided the original work is properly cited.

Peripheral neuropathy is a common and severe dose-limiting side effect of the chemotherapy agent, bortezomib, in multiple myeloma patients. Treatment with narcotics, antidepressants, and anticonvulsants has limited response and potential significant side effects. Acupuncture has been reported to be effective in treating diabetic neuropathy and chemo-induced peripheral neuropathy. There has not been report on the effect of acupuncture in treating bortezomib-induced peripheral neuropathy specifically. Here, we report a successful case of using acupuncture to relieve bortezomib-induced peripheral neuropathy symptoms.

\section{Introduction}

Peripheral neuropathy in patients with multiple myeloma occurs as both disease- and treatment-related complication in the relapsed and newly diagnosed setting. Bortezomib is a novel agent in treating multiple myeloma [1]. Bortezomibinduced peripheral neuropathy (BIPN) is one of the most common and severe toxicities of bortezomib resulting in dose reduction or drug discontinuation [2]. The incidence of BIPN varies from $37 \%$ for grade 3 to $>80 \%$ for all grades [2]. Small percentage $(\sim 6 \%)$ patients can develop painful peripheral neuropathy that usually occurs acutely and takes a long time to resolve [3]. BIPN usually occurs within the first 5 cycles of bortezomib and may resolve between 3 to 48 months after the discontinuation of the drug [2]. Major risk factors for BIPN are cumulative dose of bortezomib and pre-existing peripheral neuropathy from other causes such as diabetes or multiple myeloma. Only $31 \%(11 / 35)$ of patients experiencing grade $3 \mathrm{BIPN}$ had complete symptoms resolution after discontinuing bortezomib [4]. Prospective clinical trial with nerve conduction study suggests that BIPN is a length-dependent, sensory, axonal, large-fiber polyneuropathy [5]. Little is known about the mechanism of
BIPN. Animal studies suggest that BIPN may be the result of mild to moderate pathological changes in Schwann cells and myelin, axonal degeneration, and dorsal root ganglia neuron changes [6]. The dorsal root ganglial neuronal cell body changes may result from proteasome inhibitor such as bortezomib, which induces chromatolysis of dorsal root ganglial neurons and causes cytoplasmic accumulation of eosinophilic material [6]. Neurophysiological studies showed that bortezomib induced changes in all three major primary afferent fibers: $\mathrm{A} \beta, \mathrm{A} \delta$, and $\mathrm{C}[7]$.

The clinical characteristics of BIPN are consistent with dysfunction of sensory nerves causing spontaneous pain, paresthesia (tingling, numbness), hyperalgesia (increase sensitivity to painful stimuli), allodynia (hypersensitivity to non-painful stimuli), and decreased physical activity [8]. Patients may experience neuropathic pain in the stocking and glove distribution with an average pain score being 7.8 out of 10 [7]. They may have distal sensory loss, with the loss in lower extremities being worse than the upper extremities. They may have decreased deep tendon reflexes and changes in proprioception that affects daily activities. In addition, up to $10 \%$ of patients with BIPN experience grade 1-3 motor neuropathy [2]. The treatment for BIPN has been limited 
to symptom management with narcotics, antidepressants, anticonvulsants, and vitamins [2]. Studies suggest that such analgesic regimens usually only produce modest pain relief and are associated with side effects such as sedation, dry mouth, and constipation $[2,3,7]$. Therefore, more effective and less toxic treatments for BIPN are needed.

The efficacy of acupuncture for treating diabetic peripheral neuropathy and HIV-related peripheral neuropathy has been suggested in a number of clinical trials [9-16]. There have been a number of clinical trials showing the effect of acupuncture in reducing neuropathic pain in cancer patients $[17,18]$. One clinical trial showed the effectiveness of auricular acupuncture in treating cancer treatment induced neuropathy [18]. A case series suggested potential efficacy of body acupuncture in treating patients with chemotherapyinduced peripheral neuropathy (CIPN) [19]. To date, there has not been any clinical trial to study the effect of acupuncture in alleviating BIPN symptoms [8]. Here, we report a successful case of using acupuncture to relieve BIPN symptoms.

\section{Case Report}

A 48-year-old African-American man with multiple myeloma presented with multiple bony lesions. Past medical history was significant for hypertension and hyperlipidemia. He was a bus driver. He received induction therapy with bortezomib $1.3 \mathrm{mg} / \mathrm{m}^{2}$ IV on days $1,4,8$, and 11 , cyclophosphamide $300 \mathrm{mg} / \mathrm{m}^{2} \mathrm{IV}$ on days 1 and 8 , and dexamethasone $40 \mathrm{mg}$ PO on days of bortezomib. Cycles were repeated every 21 days. After three cycles, he achieved partial remission and underwent autologous peripheral blood stem cell transplantation. He then achieved complete remission. After he finished the third cycle of bortezomib, he developed BIPN with tingling, numbness, and burning pain of feet below the ankle despite being off bortezomib therapy and relatively low cumulative dose of the drug. His symptoms worsened over the next four months to the point that he was not able to stand or walk normally because of the severe pain. He was started on neurontin in escalating doses but was unable to tolerate doses over $1200 \mathrm{mg}$ daily due to side effects, mostly dizziness. He was given morphine sulphate (MS) contin $15 \mathrm{mg}$ PO twice daily with oxycodone $5 \mathrm{mg}$ PO every 4 to 6 hours for breakthrough pain with no significant improvement. Pregabalin $75 \mathrm{mg}$ PO twice daily was added without significant pain relief. His BIPN symptoms persisted with pain, tingling, numbness, and poor quality of life from the pain and pain medications. In addition, he was unable to drive because of pain and side effects of his pain medications. He was referred for acupuncture treatment.

During the patient's initial visit he had significant paresthesia/in bilateral lower extremities. No autonomic symptoms were reported. His self-reported overall pain score was 8 out of 10 on a 11-point visual analog scale (VAS). Neurological exam showed reduced sensation to light touch, pin, and vibration in distal part of his feet and absent ankle reflexes bilaterally. Muscle strength was five out of 5 throughout with limited lower extremity movement because of severe pain.

The patient was treated with an acupuncture protocol, which includes bilateral ear points: shen men, point zero, two
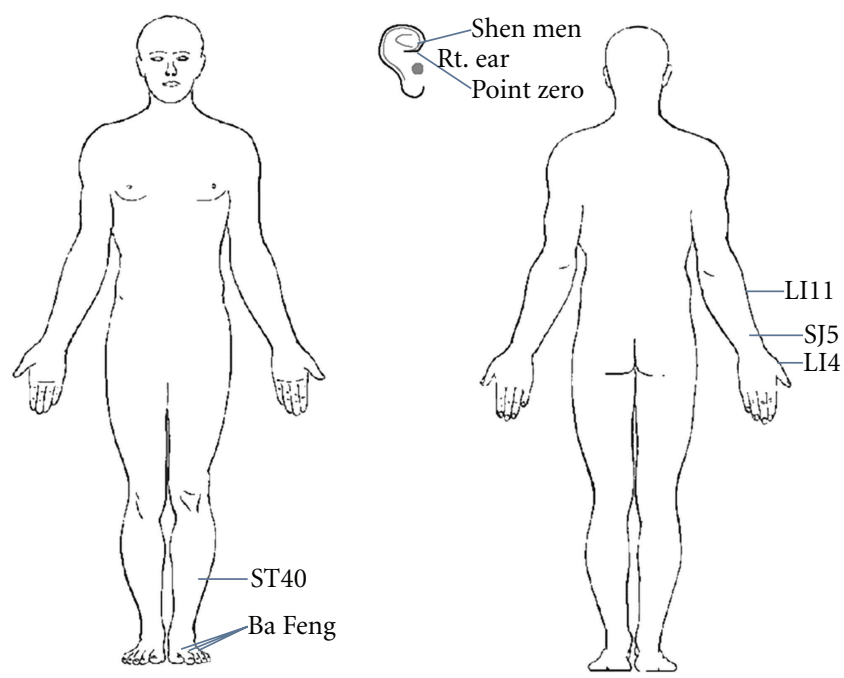

Figure 1: Acupuncture protocol to treat bortezomib-induced peripheral neuropathy.

additional auricular acupuncture point where electrodermal signal was detected [18], and bilateral body acupuncture points: LI4, SJ5, LI1 1, ST40, and a set of extraordinary points, Ba Feng (Figure 1). For auricular points, the electrodermal signal was detected through a hand-held auricular acupoint finder (the Pointer-Excell II) where a buzzing sound went off when the electrodermal signal is detected. He was treated once per week for six treatments. He noted that the initial 2 acupuncture sessions gave him pain relief for only a few hours. But the subsequent acupuncture treatments gave him progressively longer pain relief. At the end of his six acupuncture treatments, his self-reported overall pain score went down from 8 out of 10 to 2 out of 10 on VAS. His use of breakthrough pain medication (oxycodone) decreased from 20 to $30 \mathrm{mg}$ daily to 5 to $10 \mathrm{mg}$ daily. He no longer had difficulty walking and standing. He continued acupuncture every other week for four treatments, then once every three weeks for two treatments, and finished with once per month for two treatments. At the end of his 14 acupuncture treatments, he stopped taking MS contin and Oxycodone and was able to go back to work to drive buses. He has been followed every three months and had no recurrent symptoms at last followup, 1 year after the initiation of his symptoms. Throughout his 14 acupuncture treatments, no adverse events related to acupuncture were noted.

\section{Discussion}

This is the first case report on the use of acupuncture in treating multiple myeloma patients with BIPN. Our report suggests that acupuncture is a treatment option for patients experiencing BIPN. Fourteen acupuncture treatments helped the patient to have significant less neuropathic pain, reduced requirement for narcotics, and improved function with minimal side effects.

The acupuncture protocol was developed from our clinical experience and prior chemo-induced peripheral 
neuropathy acupuncture research $[18,19]$. One randomized, blinded clinical trial showed the effectiveness of auricular acupuncture in treating cancer therapy induced neuropathy [18]. The patients were randomized into one of three arms. One arm received real auricular acupuncture embedded at real ear acupuncture points, which is defined as points where the electrodermal signal is being detected. The other two arms were sham control arms: one received real auricular acupuncture at the placebo points and one received sham acupuncture through auricular seeds at the placebo points [18]. All patients received two courses of real or sham auricular acupuncture in two consecutive months. The needles were left in place until they fell out or were removed at a followup appointment. Their pain intensity measured by VAS at the end of the second month was used to measure the treatment efficacy. This study showed that in the group that received real acupuncture, pain intensity decreased by $36 \%$ at the end of 2 months when compared with baseline, whereas it only decreased by $2 \%$ in the placebo groups $(P<.0001)$ [18]. Moreover, a case series suggested potential efficacy of body acupuncture in treating patients with chemotherapyinduced peripheral neuropathy (CIPN) [19]. Five patients with greater than world health organization grade II CIPN were treated with 6 weekly acupuncture treatments followed by 4 weeks of rest and 6 additional weekly acupuncture treatments. All patients but one finished all 12 acupuncture treatments. All five patients reported improvement in pain, tingling, and numbness. Pain scores decreased from $6-9 / 10$ to $2-3 / 10$ after acupuncture treatments. All patients had reduced analgesic medication intake. Four out of five patients reported persistent symptoms relief at the 6 month followup [19].

We observed similar effect in our case report to those aforementioned reports, which is that patient with CIPN responded to acupuncture treatments. Our case suggests that acupuncture may be effective in relieving BIPN symptoms. Although studies have not been able to fully explain the mechanism of acupuncture, it has been proposed that acupuncture works through its effect on neurotransmitters and neurohormones [20-23]. Animal research suggests that acupuncture accomplishes its anesthesia effect by stimulating nerves in the muscle, which then relay the signal to the spinal cord, midbrain, and hypothalamus-pituitary system, which then lead to the release of neurotransmitters and hormones, that is, endorphins and enkephalins [24-26]. Other mechanisms such as activation of descending pain inhibiting pathways, deactivation of the limbic system, cortical cerebral vasodilation causing release of neuropeptide, and inhibition of the release of inflammatory factors have also been suggested to explain the effect of acupuncture analgesia [27-31]. Recent study also showed that electroacupuncture alleviates bone cancer pain by suppressing spinal expression of interleukin- $1 \beta$ in glia cells that are involved in the spinal transmission and processing of noxious inputs from the peripheral sites and facilitates pain [32]. It is perceivable that acupuncture reduces BIPN symptoms by suppressing activities of glial cell and stimulating neurohormonal pathways, which increases endorphins release and reduces proinflammatory cytokines. Further study needs to be conducted to explore the role of acupuncture in helping patients suffering from BIPN and its mechanisms of action.

Our case report is limited in a couple of ways. First, the patient's relief of peripheral neuropathy symptoms may be due to a spontaneous remission rather than the effect of acupuncture. However, based on our clinical experience, once BIPN becomes so severe that it interferes with daily activities, it rarely resolves by itself, and even so, it usually takes a long time (months to years) to do so. Second, the clinical improvement in this patient was primarily documented by subjective end point, the VAS score. Changes in objective end points such as detailed neurological exam and nerve conduction study will help understand how acupuncture worked in reducing BIPN. This case report is the beginning of our endeavor to investigate the role of acupuncture in relieving BIPN. We plan to incorporate more objective measurements such as nerve conduction study into future studies on this topic.

\section{Acknowledgments}

T. Bao is a Paul Calabresi scholar (K12 CA126849 A). R. Zhang and L. Lao are supported by Grant no. AT004113 from the National Center for Complementary and Alternative Medicine (NCCAM) at the National Institutes of Health. The content is solely the responsibility of the authors and does not necessarily represent the official views of NCCAM.

\section{References}

[1] J. San Miguel, J. Bladé, M. Boccadoro et al., "A practical update on the use of bortezomib in the management of multiple myeloma," Oncologist, vol. 11, no. 1, pp. 51-61, 2006.

[2] A. A. Argyriou, G. Iconomou, and H. P. Kalofonos, "Bortezomib-induced peripheral neuropathy in multiple myeloma: a comprehensive review of the literature," Blood, vol. 112, no. 5, pp. 1593-1599, 2008.

[3] A. Badros, O. Goloubeva, J. S. Dalal et al., "Neurotoxicity of bortezomib therapy in multiple myeloma: a single-center experience and review of the literature," Cancer, vol. 110, no. 5, pp. 1042-1049, 2007.

[4] P. G. Richardson, H. Briemberg, S. Jagannath et al., "Frequency, characteristics, and reversibility of peripheral neuropathy during treatment of advanced multiple myeloma with bortezomib," Journal of Clinical Oncology, vol. 24, no. 19, pp. 3113-3120, 2006.

[5] V. Chaudhry, D. R. Cornblath, M. Polydefkis, A. Ferguson, and I. Borrello, "Characteristics of bortezomib- and thalidomideinduced peripheral neuropathy: research report," Journal of the Peripheral Nervous System, vol. 13, no. 4, pp. 275-282, 2008.

[6] G. Cavaletti, A. Gilardini, A. Canta et al., "Bortezomibinduced peripheral neurotoxicity: a neurophysiological and pathological study in the rat," Experimental Neurology, vol. 204, no. 1, pp. 317-325, 2007.

[7] J. P. Cata, H. R. Weng, A. W. Burton, H. Villareal, S. Giralt, and P. M. Dougherty, "Quantitative sensory findings in patients with bortezomib-induced pain," Journal of Pain, vol. 8, no. 4, pp. 296-306, 2007.

[8] Y. Zhou, M. K. Garcia, D. Z. Chang et al., "Multiple myeloma, painful neuropathy, acupuncture?" American Journal of Clinical Oncology, vol. 32, no. 3, pp. 319-325, 2009. 
[9] B. B. Abuaisha, J. B. Costanzi, and A. J. M. Boulton, "Acupuncture for the treatment of chronic painful peripheral diabetic neuropathy: a long-term study," Diabetes Research and Clinical Practice, vol. 39, no. 2, pp. 115-121, 1998.

[10] C. Zhang, Y. X. Ma, and Y. E. Yan, "Clinical effects of acupuncture for diabetic peripheral neuropathy," Journal of Traditional Chinese Medicine, vol. 30, no. 1, pp. 13-14, 2010.

[11] J. L. Zhao and Z. R. Li, "Clinical observation on mild-warm moxibustion for treatment of diabetic peripheral neuropathy," Zhongguo Zhen Jiu, vol. 28, no. 1, pp. 13-16, 2008.

[12] A. C. Ahn, T. Bennani, R. Freeman, O. Hamdy, and T. J. Kaptchuk, "Two styles of acupuncture for treating painful diabetic neuropathy - a pilot randomised control trial," Acupuncture in Medicine, vol. 25, no. 1-2, pp. 11-17, 2007.

[13] H. L. Zhao, X. Gao, and Y. B. Gao, "Clinical observation on effect of acupuncture in treating diabetic peripheral neuropathy," Zhongguo Zhong Xi Yi Jie He Za Zhi, vol. 27, no. 4, pp. 312-314, 2007.

[14] H. Jiang, K. Shi, X. Li, W. Zhou, and Y. Cao, "Clinical study on the wrist-ankle acupuncture treatment for 30 cases of diabetic peripheral neuritis," Journal of Traditional Chinese Medicine, vol. 26, no. 1, pp. 8-12, 2006.

[15] K. D. Phillips, W. D. Skelton, and G. A. Hand, "Effect of acupuncture administered in a group setting on pain and subjective peripheral neuropathy in persons with human immunodeficiency virus disease," Journal of Alternative and Complementary Medicine, vol. 10, no. 3, pp. 449-455, 2004.

[16] M. L. A. Galantino, S. T. Eke-Okoro, T. W. Findley, and D. Condoluci, "Use of noninvasive electroacupuncture for the treatment of HIV-related peripheral neuropathy: a pilot study," Journal of Alternative and Complementary Medicine, vol. 5, no. 2, pp. 135-142, 1999.

[17] O. Minton and I. J. Higginson, "Electroacupuncture as an adjunctive treatment to control europathic pain in patients with cancer," Journal of Pain and Symptom Management, vol. 33, no. 2, pp. 115-117, 2007.

[18] D. Alimi, C. Rubino, E. Pichard-Léandri, S. Fermand-Brulé, M. L. Dubreuil-Lemaire, and C. Hill, "Analgesic effect of auricular acupuncture for cancer pain: a randomized, blinded, controlled trial," Journal of Clinical Oncology, vol. 21, no. 22, pp. 4120-4126, 2003.

[19] R. Wong and S. Sagar, "Acupuncture treatment for chemotherapy-induced peripheral neuropathy-a case series," Acupuncture in Medicine, vol. 24, no. 2, pp. 87-91, 2006.

[20] C. Takeshire, "Mechanism of acupuncture analgesia based on animal experiments," in Scientific Bases of Acupuncture, B. Pomerantz and G. Stux, Eds., Springer, Berlin, Germany, 1989.

[21] B. Y. Lee, P. J. LaRiccia, and A. B. Newberg, "Acupuncture in theory and practice," Hospital Physician, vol. 40, pp. 11-18, 2004.

[22] L. Lao, R. X. Zhang, G. Zhang, X. Wang, B. M. Berman, and K. Ren, "A parametric study of electroacupuncture on persistent hyperalgesia and Fos protein expression in rats," Brain Research, vol. 1020, no. 1-2, pp. 18-29, 2004.

[23] R. X. Zhang, L. Lao, L. Wang et al., "Involvement of opioid receptors in electroacupuncture-produced anti-hyperalgesia in rats with peripheral inflammation," Brain Research, vol. 1020, no. 1-2, pp. 12-17, 2004.

[24] J. S. Han, "Acupuncture: neuropeptide release produced by electrical stimulation of different frequencies," Trends in Neurosciences, vol. 26, no. 1, pp. 17-22, 2003.
[25] B. Berman, "A 60-year-old woman considering acupuncture for knee pain," Journal of the American Medical Association, vol. 297, no. 15, pp. 1697-1707, 2007.

[26] A. Li, L. Lao, Y. I. Wang et al., "Electroacupuncture activates corticotrophin-releasing hormone-containing neurons in the paraventricular nucleus of the hypothalammus to alleviate edema in a rat model of inflammation," BMC Complementary and Alternative Medicine, vol. 8, article 20, 2008.

[27] K. K. S. Hui, J. Liu, O. Marina et al., "The integrated response of the human cerebro-cerebellar and limbic systems to acupuncture stimulation at ST 36 as evidenced by fMRI," NeuroImage, vol. 27, no. 3, pp. 479-496, 2005.

[28] G. Jansen, T. Lundeberg, J. Kjartansson, and U. E. Samuelson, "Acupuncture and sensory neuropeptides increase cutaneous blood flow in rats," Neuroscience Letters, vol. 97, no. 3, pp. 305309, 1989.

[29] H. Kashiba and Y. Ueda, "Acupuncture to the skin induces release of substance $\mathrm{P}$ and calcitonin gene-related peptide from peripheral terminals of primary sensory neurons in the rat," American Journal of Chinese Medicine, vol. 19, no. 3-4, pp. 189-197, 1991.

[30] R. J. Leong and B. Chernow, "The effects of acupuncture on operative pain and the hormonal responses to stress," International Anesthesiology Clinics, vol. 26, no. 3, pp. 213-217, 1988.

[31] A. Li, Y. I. Wang, J. Xin et al., "Electroacupuncture suppresses hyperalgesia and spinal fos expression by activating the descending inhibitory system," Brain Research, vol. 1186, no. 1, pp. 171-179, 2007.

[32] R. X. Zhang, A. Li, B. Liu et al., "Electroacupuncture attenuates bone cancer pain and inhibits spinal interleukin- $1 \beta$ expression in a rat model," Anesthesia and Analgesia, vol. 105, no. 5, pp. 1482-1488, 2007. 


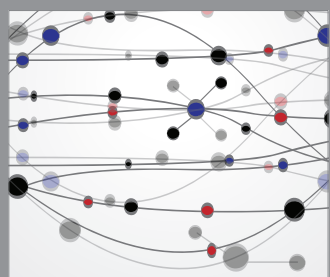

The Scientific World Journal
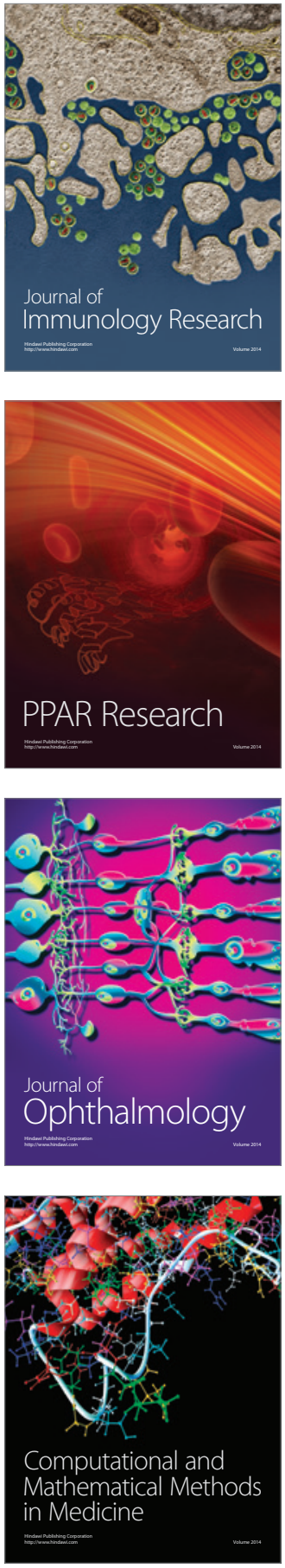

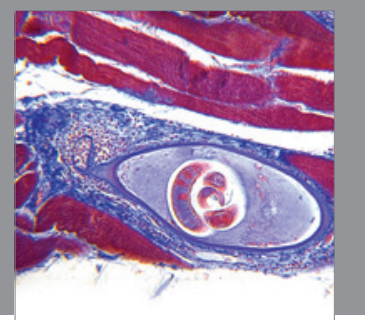

Gastroenterology

Research and Practice
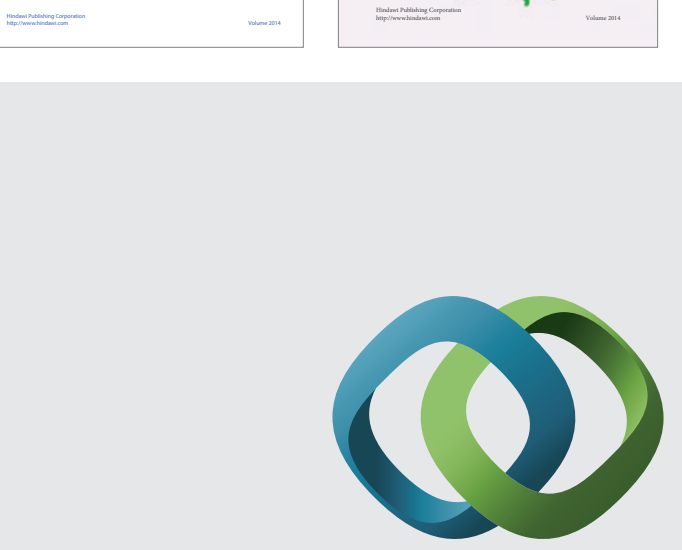

\section{Hindawi}

Submit your manuscripts at

http://www.hindawi.com
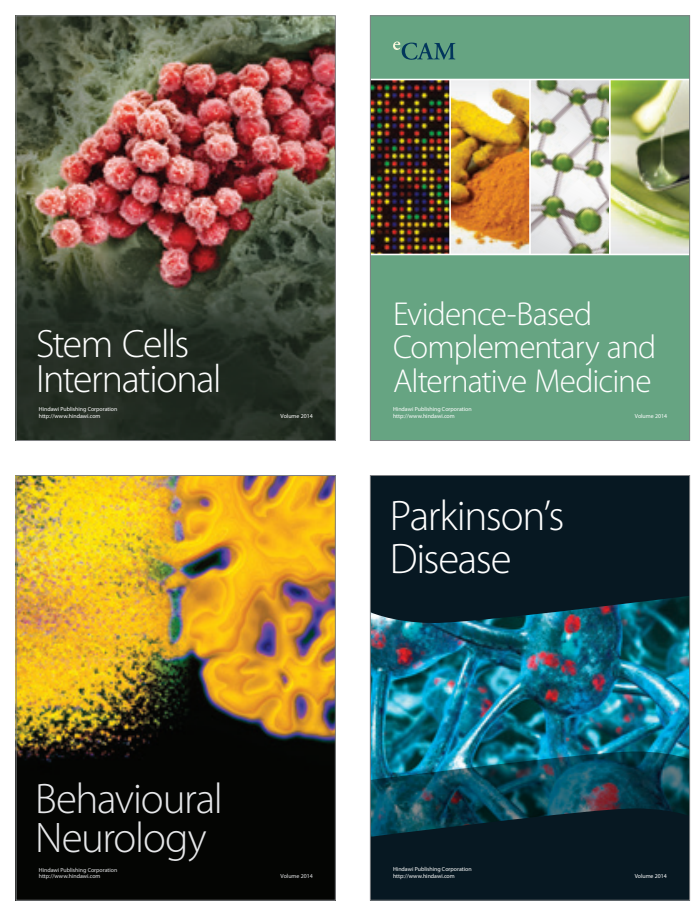

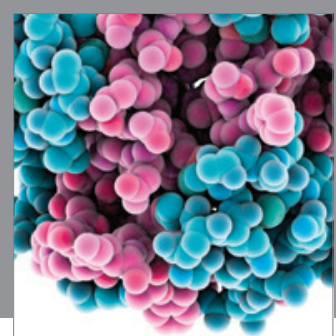

Journal of
Diabetes Research

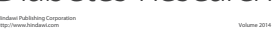

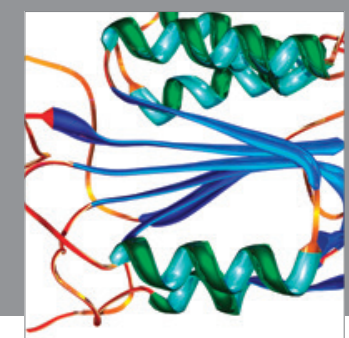

Disease Markers
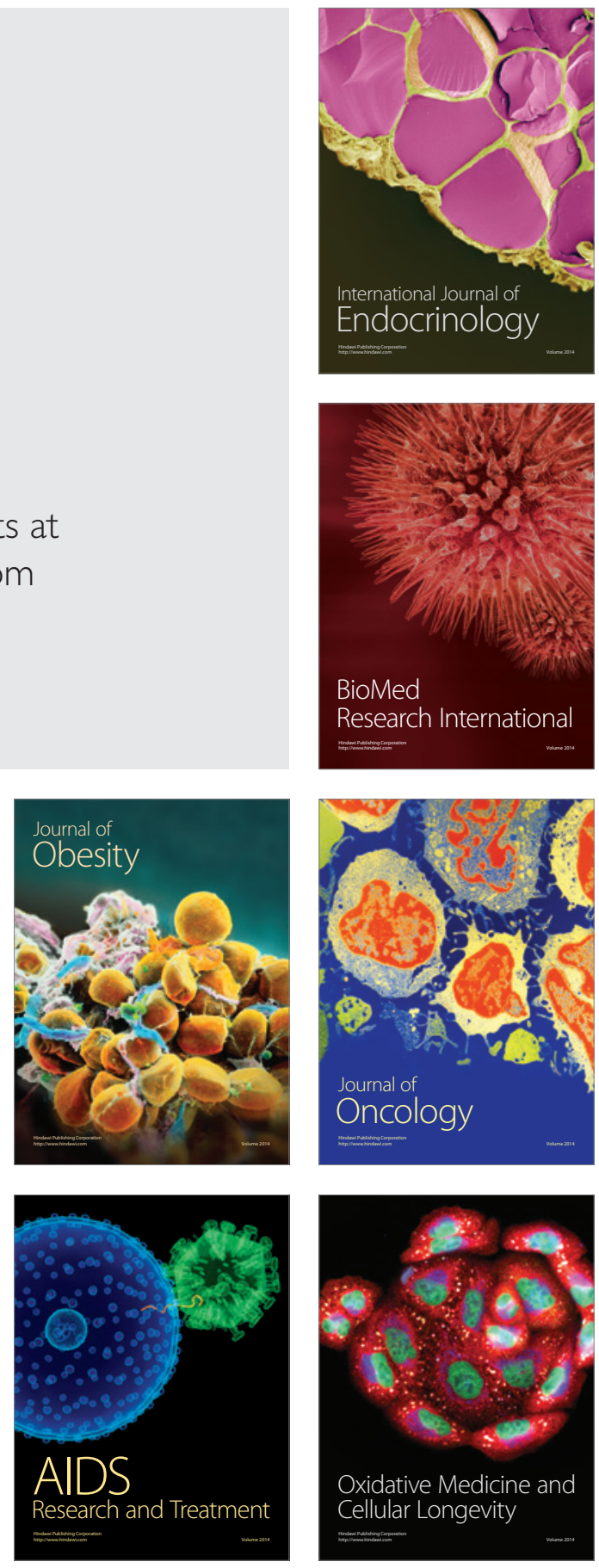